\title{
Line asymmetry of solar p-modes: Reversal of asymmetry in intensity power spectra
}

\author{
Pawan Kumar and Sarbani Basu \\ Institute for Advanced Study, Olden Lane, Princeton, NJ 08540, U. S. A.
}

Received — 


\begin{abstract}
The sense of line asymmetry of solar p-modes in the intensity power spectra is observed to be opposite of that seen in the velocity power spectra. Theoretical calculations provide a good understanding and fit to the observed velocity power spectra whereas the reverse sense of asymmetry in the intensity power spectrum has been poorly understood. We show that when turbulent eddies arrive at the top of the convection zone they give rise to an observable intensity fluctuation which is correlated with the oscillation they generate, thereby affecting the shape of the line in the p-mode power spectra and reversing the sense of asymmetry (this point was recognized by Nigam et al. and Roxburgh \& Vorontsov). The addition of the correlated noise displaces the frequencies of peaks in the power spectrum. Depending on the amplitude of the noise source the shift in the position of the peak can be substantially larger than the frequency shift in the velocity power spectra. In neither case are the peak frequencies precisely equal to the eigenfrequencies of p-modes. We suggest two observations which can provide a test of the model discussed here.
\end{abstract}

Subject headings: Sun: oscillations; convection; turbulence 


\section{Introduction}

Duvall et al. (1993) found that solar p-mode line profiles are not exactly Lorentzian in shape but are somewhat asymmetric about the peak of the power. They showed that the power in their observed surface velocity spectra fell off more slowly at the low frequency side of the peak compared to the high frequency side of the peak. They also claimed that the sense of asymmetry is opposite in the intensity power spectra i.e. there is more power at the high frequency side of the peak compared to the low frequency side. The results of Duvall et al. have been recently confirmed by a number of independent observations.

Theoretical calculations have little difficulty in reproducing the observed velocity power spectrum. According to the simplest models one expects the shape of velocity and intensity power spectra to be almost identical, as long as both of these are observed at the same place in the solar atmosphere, since the velocity and temperature eigenfunctions of a mode are linearly related to each other. Rast \& Bogdan (1998) point out that nonadiabatic effects are unlikely to modify this relation. Nor does it appear that the process of the formation of absorption lines in the optical spectrum could cause the asymmetry of intensity power spectrum to be reversed (Sasselov, personal communication). It is therefore puzzling that the intensity power spectra are observed to be very different from the velocity spectra. Nigam et al. (1998) suggested that correlated noise added to p-mode oscillation amplitude affects the shape of the observed spectrum and could be responsible for the difference between velocity and intensity power spectra; they suggested that granules could provide the correlation. Roxburgh \& Vorontsov (1997) suggested that velocity variation associated with acoustic sources, i.e., the turbulent eddies, are observed when eddies arrive at the top of the convection zone and this variation should be correlated with p-mode oscillations. Roxburgh \& Vorontsov chose, arbitrarily, the relative amplitude of the velocity variation associated with oscillation and with the noise source to be of order unity and considered 
them to be almost perfectly correlated in order to produce the reversal of line asymmetry.

We calculate the relative contribution of turbulent eddies to the observed flux variation and its correlation with p-mode oscillation in a self consistent manner and show that this process explains the observed reversal of asymmetry in the intensity power spectra. The calculations are presented in section 2. The conclusion and some predictions are contained in section 3 .

\section{Intensity oscillation and line asymmetry}

We first consider a simple one dimensional square well potential model, same as that considered in Abrams \& Kumar 1996, to calculate the power spectrum of p-mode oscillations and include the contribution of fluctuating sources, which excite these oscillations, to the observed flux variation amplitude:

$$
\frac{d^{2} \psi_{\omega}}{d r^{2}}+i 2 \omega \gamma \psi_{\omega}+\left[\omega^{2}-V(r)\right] \psi_{\omega}=S_{\omega} \delta\left(r-r_{s}\right)
$$

where $\psi_{\omega}$ is the temperature perturbation function, $S_{\omega}$ is the quadrupole source amplitude, $\gamma$ is the damping constant which is taken to be independent of $r$, and $V(r)$ is a simplified effective potential constructed to describe the solar p-modes: $V=\infty$ for $r \leq 0$, for $0<r<a$ the potential is zero, and $V(r)=\alpha^{2}$, a constant, for $r \geq a$; $a$ is the sound travel time from the lower to the upper turning point of a given mode and $\alpha$ is the acoustic cutoff frequency at the temperature minimum. The observed flux variation is roughly proportional to the Lagrangian temperature perturbation at the place in the atmosphere where lines are formed'.

\footnotetext{
${ }^{1} \mathrm{~A}$ perturbation of the convective flux by oscillations could however modify the relation between the observed flux variation and the Lagrangian temperature perturbation.
} 
For sources lying in the evanescent region i.e. $r_{s} \geq a$, the amplitude of the temperature variation observed at point $r_{o}$ can be easily calculated by solving Eq. (1) and is given by

$$
T_{\omega}\left(r_{o}\right)=S_{\omega} G_{\omega}\left(r_{s}, r_{o}\right)+\frac{S_{\omega} \beta}{\omega_{2}} \exp (i \phi-\eta)
$$

where

$$
G_{\omega}\left(r_{s}, r_{o}\right)=-\frac{\exp \left[-\omega_{2}(r-a)\right]}{\omega_{2}}\left[\frac{\omega_{2} \sin \left(\omega_{1} a\right) \cosh \left(\omega_{2} \delta r\right)+\omega_{1} \cos \left(\omega_{1} a\right) \sinh \left(\omega_{2} \delta r\right)}{\omega_{2} \sin \omega_{1} a+\omega_{1} \cos \omega_{1} a}\right],
$$

is the Green's function for Eq. (1), $\omega_{1}=\left(\omega^{2}+i 2 \gamma \omega\right)^{1 / 2}, \omega_{2}=\left(\alpha^{2}-\omega^{2}-i 2 \gamma \omega\right)^{1 / 2}$, $\delta r=r_{s}-a$, and $\eta$ is the optical depth between $r_{o}$, the point where the observation is made, and the top of the convection zone. The dimensionless parameter $\beta$ is the ratio of the observed amplitude of the flux variation of an eddy to that of the oscillation, and $\phi$ is its phase difference with respect to the mode it excites at radius $r_{o}$.

The real and the imaginary parts of the Green's function have very different shapes as shown in fig. 1, and this enables a correlated noise source to modify the observed shape of the power spectrum as discussed below.

In the absence of the contribution of the noise source to the observed flux variation, i.e. when $\beta=0$, the shape of the power spectrum is shown in the left panel of Fig. 2. This case should correspond to the velocity power spectrum of solar p-modes since the random component of the velocity field in the solar atmosphere - where optical observations are made - is believed to be uncorrelated with the turbulent eddies in the convection zone which excite p-mode oscillations. The asymmetry in our calculated spectrum does in fact look very much like that seen in the velocity power spectra of solar p-modes.

Unlike the velocity variations, the flux variation associated with turbulent eddies are observed when the eddies arrive at the top of the convection zone. These variations are correlated with oscillations these eddies generate; this correlation is parameterized above 
by $\beta$ and $\phi$. The addition of this 'noise' term to the oscillatory signal modifies the relative contribution of the real and the imaginary parts of the Green's function to the observed intensity spectrum (which is proportional to $\left.\left|T_{\omega}\left(r_{o}\right)\right|^{2}\right)$ and thereby modifies the shape of

the p-mode lines in the intensity observations (see Fig. 2, right panel). We find the reversal of asymmetry over a wide range of $\beta$ and $\phi$ as shown in fig. 3 ; these line profiles are similar to the observed reversal of asymmetry seen in the solar intensity data. Note that the background in the intensity spectrum is significantly non-zero, and in fact if this model is correct we expect the reversal of asymmetry to disappear when the background in the spectrum vanishes.

The addition of the correlated noise source to the observed flux variation significantly modifies frequencies of peaks in the power spectrum as is clearly seen in Fig. 3. A change in the amplitude of the noise source, as quantified by the dimensionless parameter $\beta$, from 0.5 to 2 shifts the peak frequency of $2.4 \mathrm{mHz}$ mode by about $0.4 \mu \mathrm{Hz}$. The peak frequencies in the intensity power spectra are different from the peaks in the velocity spectra and neither of these are the true eigenfrequencies of p-modes. The frequency shift in the intensity spectra depends on the amplitude of the background in addition to the effects that cause shift in the velocity spectra (cf. Abrams \& Kumar 1996, Christensen-Dalsgaard et al. 1998), and so it is safer to use the velocity spectra to determine the true eigenfrequencies of solar p-modes.

We calculate the parameters $\beta$ and $\phi$ for a realistic model below.

\subsection{Relation between wave and source amplitudes}

We consider a plane parallel atmosphere which sits in a constant gravitational field, g, and consists of two semi-infinite layers, the lower layer being adiabatic and polytropic, 
and the upper isothermal. The pressure, $p$, density, $\rho$, and temperature, $T$, are continuous across the interface between the two layers. In the lower layer the adiabatic and polytropic indices are related by $\gamma=1+1 / m$. The $z$ coordinate measures depth below the level at which the adiabatic layer would terminate in the absence of the isothermal layer. In the adiabatic layer the thermodynamic variables have a power law dependence on the depth $z$ e.g. $p=p_{t}\left(z / z_{t}\right)^{m+1}, \rho=\rho_{t}\left(z / z_{t}\right)^{m}$, the sound speed $c^{2}=g z / m$, and the scale height $H=z /(m+1)$, where the subscript $t$ denotes quantities evaluated at the top of the adiabatic layer. In the isothermal atmosphere $p$ and $\rho$ are proportional to $\exp \left(z / H_{i}\right)$.

Following Goldreich and Kumar (1990), hereafter referred to as GK, we use $Q=P_{1} / \rho$ as the wave variable, where $P_{1}$ is the Eulerian pressure perturbation. The inhomogeneous wave equation in the adiabatic atmosphere is

$$
\frac{d^{2} Q}{d z^{2}}+\frac{g}{c^{2}} \frac{d Q}{d z}+-\frac{1}{c^{2}} \frac{\partial^{2} Q}{\partial t^{2}}-k_{h}^{2} Q=-\frac{\partial^{2}}{\partial t^{2}}\left(\frac{s}{c_{p}}\right)-\frac{g}{\rho} \frac{\partial}{\partial z}\left(\frac{\rho s}{c_{p}}\right) \equiv S,
$$

where $s$ is the Eulerian entropy perturbation associated with the turbulent convection. The source term $S$ consists of a monopole and a dipole piece which arise as a result of volume change due to fluctuation in entropy and buoyancy force variation associated with this volume change respectively. For simplicity we have not included the Reynold's stress source term associated with fluctuating internal stresses in the convection zone. Inclusion of Reynold's stress makes the algebra considerably more tedious but does not modify the main results of this section in any essential way.

We expand the enthalpy wave function $Q$ in terms of the normal modes, $Q_{\alpha}(z)$, which are normalized to unit energy,

\footnotetext{
${ }^{2}$ GK showed that the monopole and the dipole source terms in $S$ cancel leaving the remainder which has magnitude of same order as the quadrupole Reynold's term.
} 


$$
Q=\frac{1}{\sqrt{2 \mathcal{A}}} \sum_{\alpha}\left[A_{\alpha} Q_{\alpha} \exp \left(-i \omega t+i \mathbf{k}_{\mathbf{h}} \cdot \mathbf{x}\right)+A_{\alpha}^{*} Q_{\alpha}^{*} \exp \left(i \omega t-i \mathbf{k}_{\mathbf{h}} \cdot \mathbf{x}\right)\right],
$$

where $\mathcal{A}$ is the horizontal cross section area of the atmosphere, and $\mathbf{k}_{\mathbf{h}}$ is the horizontal wave vector. Substituting this expansion into equation (4) and multiplying both sides with $Q_{\alpha}^{*} \exp \left(i \omega t-i \mathbf{k}_{\mathbf{h}} \cdot \mathbf{x}\right)$, and integrating over space and time, we obtain

$$
A_{\alpha}(t)=\frac{1}{2 i \omega \mathcal{A}^{1 / 2}} \int_{-\infty}^{t} d t \int d^{3} x Q_{\alpha}^{*} S \exp \left(i \omega t-i \mathbf{k}_{\mathbf{h}} \cdot \mathbf{x}\right) .
$$

Using the wave equation (4) this can be rewritten as

$$
A_{\alpha}(t) \approx-\frac{1}{2 i \omega \mathcal{A}^{1 / 2}} \int_{-\infty}^{t} d t \int d^{3} x \frac{\rho c^{2} s}{c_{p}} \frac{\partial^{2} Q_{\alpha}^{*}}{\partial z^{2}} \exp \left(i \omega t-i \mathbf{k}_{\mathbf{h}} \cdot \mathbf{x}\right) .
$$

The change to mode amplitude, $\delta A_{\alpha}$, due to a single eddy of size $h$ over its lifetime $\tau_{h}$, which is located at $\mathbf{x}_{\mathbf{0}}$ and lived at time $t_{0}$, can be easily estimated from the above equation and is given by

$$
\delta A_{\alpha}(t) \approx-\frac{\rho c^{2} h^{3} \tau_{h} s_{h}\left(t_{0}\right)}{2 i \omega c_{p} \mathcal{A}^{1 / 2}} \frac{\partial^{2} Q_{\alpha}^{*}}{\partial z^{2}} \exp \left[i \omega_{\alpha} t_{0}-i \mathbf{k}_{\mathbf{h}} \cdot \mathbf{x}_{\mathbf{0}}-\gamma_{\alpha}\left(t-t_{0}\right)+i \phi_{1}\right],
$$

where the phase $\phi_{1}$ depends on the temporal and spacial properties of the eddy, and the factor $\exp \left[-\gamma_{\alpha}\left(t-t_{0}\right)\right]$ has been multiplied to model wave damping that causes the change to mode amplitude to decrease with time for $t \geq t_{0}$. GK showed that the resonant scale height size eddies are most efficient at exciting low frequency modes (modes of frequency less than about $3 \mathrm{mHz})$; for these eddies $h \sim H$ and $\tau_{h} \sim \omega_{\alpha}^{-1}$. The depth $\left(z_{\omega}\right)$ of these eddies below the photosphere, or the isothermal atmosphere in our model, can be estimated from the constancy of the convective flux $\rho v^{3}$

$$
z_{\omega} \sim z_{t}\left[\frac{\mathcal{M}_{t} \omega_{a c}}{\omega}\right]^{3 /(m+3)}
$$


where $\mathcal{M}_{t}$ is the Mach number of the turbulent convection at the top of the convection zone, and $\omega_{a c}$ is the acoustic cut off frequency in the isothermal atmosphere.

The change to the enthalpy perturbation associated with a mode $\alpha, \delta Q_{\alpha}\left(z_{o}\right)$, in the isothermal atmosphere at location $z_{o}$ due to the eddy is given by

$$
\delta Q_{\alpha}\left(z_{o}, t\right)=\frac{\delta A_{\alpha} Q_{\alpha}\left(z_{o}\right) \exp \left(-i \omega_{\alpha} t+i \mathbf{k}_{\mathbf{h}} \cdot \mathbf{x}\right)}{(2 \mathcal{A})^{1 / 2}} .
$$

Substituting for $\delta A_{\alpha}$ from Eq. (8) we find

$\delta Q_{\alpha}\left(z_{o}, t\right)=i \frac{\rho\left(z_{\omega}\right) c^{2}\left(z_{\omega}\right) H_{\omega}^{3}}{2^{3 / 2} \mathcal{A} \omega^{2}} \frac{\delta T_{e d}\left(z_{\omega}, t_{0}\right)}{T\left(z_{\omega}\right)} Q_{\alpha}\left(z_{o}\right) \frac{\partial^{2} Q_{\alpha}^{*}}{\partial z^{2}} \exp \left[-\left(\gamma_{\alpha}+i \omega_{\alpha}\right)\left(t-t_{0}\right)+i \phi_{1}+i \mathbf{k}_{\mathbf{h}} \cdot\left(\mathbf{x}-\mathbf{x}_{0}\right)\right]$

where $\delta T_{e d}=s_{h} / c_{p}$ is the temperature fluctuation of the eddy. Low frequency p-modes are most likely excited near the top of the convection zone in a region where waves are evanescent (cf. GK). The wave function is nearly constant in the evanescent region and its derivative $\partial^{2} Q_{\alpha} / \partial z^{2} \sim\left(\omega^{4} / g^{2}\right) Q_{\alpha}$. Moreover, the normalized eigenfunction in the evanescent region is (see GK)

$$
Q_{\alpha} \sim \frac{z_{t}^{m / 2} \omega^{(m-1)} k_{h}^{1 / 2}}{\rho_{t}^{1 / 2} g^{(m-2) / 2}}
$$

Making use of these equations, the expression for $\delta Q_{\alpha}$ reduces to

$$
\delta Q_{\alpha}\left(z_{o}, t\right) \sim \frac{i k_{h} z_{\omega}^{m+3} \omega^{2 m}}{\mathcal{A} g^{m}} c_{p} \delta T_{e d}\left(z_{\omega}, t_{0}\right) \exp \left[-\left(\gamma_{\alpha}+i \omega_{\alpha}\right)\left(t-t_{0}\right)+i \mathbf{k}_{\mathbf{h}} \cdot\left(\mathbf{x}-\mathbf{x}_{\mathbf{0}}\right)+\mathbf{i} \phi_{\mathbf{1}}\right] .
$$

Finally substituting for $z_{\omega}$ from Eq. (9) and using the relation $g / z_{t} \approx \omega_{a c}^{2}$, the above equation further simplifies to 
$\delta Q_{\alpha}\left(z_{o}, t\right) \sim \frac{i k_{h} z_{t}^{3}}{\mathcal{A}} \mathcal{M}_{t}^{3}\left(\frac{\omega}{\omega_{a c}}\right)^{2 m-3} c_{p} \delta T_{e d}\left(z_{\omega}, t_{0}\right) \exp \left[-\left(\gamma_{\alpha}+i \omega_{\alpha}\right)\left(t-t_{0}\right)+i \mathbf{k}_{\mathbf{h}} \cdot\left(\mathbf{x}-\mathbf{x}_{0}\right)+i \phi_{1}\right]$

We next consider the relation between $\delta Q$ and the associated change in the temperature amplitude of the mode at the height in the atmosphere where optical observations are made. For the nearly isothermal solar atmosphere the Lagrangian temperature perturbation and the pressure perturbations are related by

$$
\Delta T=\Delta p\left(\frac{\partial T}{\partial p}\right)_{s} \approx \frac{\Delta p}{\rho c_{p}}=\frac{p_{1}+\rho g \xi_{z}}{\rho c_{p}}
$$

where $\xi_{z}$ is the radial displacement associated with the wave, and for low frequency acoustic waves it is given by (see GK for detail)

$$
\xi_{z} \approx-\frac{1}{\omega_{a c}^{2}}\left[\frac{d Q}{d z}+\frac{(\gamma-1) Q}{\gamma H_{i}}\right] \approx-\frac{Q}{H_{i} \omega_{a c}^{2}}\left[\frac{\gamma-1}{\gamma}-\frac{\omega^{2}}{4 \omega_{a c}^{2}}\right]
$$

Therefore,

$$
\Delta T \approx \frac{\omega^{2}}{\omega_{a c}^{2}} \frac{Q}{c_{p}}
$$

Substituting this into Eq. (14) we obtain the change to the Lagrangian temperature perturbation in the isothermal atmosphere as a result of wave generated by the entropy fluctuation of an eddy

$$
\Delta T_{\alpha}\left(z_{o}, \mathbf{x}_{h}, t\right) \approx i F\left(\omega, k_{h}\right) \delta T_{e d}\left(z_{\omega}, t_{0}\right) \exp \left[-\left(\gamma_{\alpha}+i \omega_{\alpha}\right)\left(t-t_{0}\right)+i \mathbf{k}_{\mathbf{h}} \cdot\left(\mathbf{x}-\mathbf{x}_{\mathbf{0}}\right)+i \phi_{1}\right]
$$

where $F\left(\omega, k_{h}\right)$ is a dimensionless function defined by 


$$
F\left(\omega, k_{h}\right)=\frac{k_{h} z_{t}^{3}}{\mathcal{A}}\left(\frac{\omega}{\omega_{a c}}\right)^{2 m-1} \mathcal{M}_{t}^{3}
$$

The total observed temperature fluctuation in the atmosphere, as seen in a line or continuum, at some location on the solar disk, consists of the contribution from oscillations and the attenuated emission from turbulent eddies at the top of the convection zone.

$$
\begin{gathered}
\delta T_{a}\left(z_{o}, \mathbf{x}_{h}, t\right)=i \sum_{\text {eddies }} F\left(\omega, k_{h}\right) \delta T_{e d}\left(z_{\omega}, \mathbf{x}_{0}, t_{0}\right) \exp \left[-\left(\gamma_{\alpha}+i \omega_{\alpha}\right)\left(t-t_{0}\right)+i \mathbf{k}_{\mathbf{h}} \cdot\left(\mathbf{x}-\mathbf{x}_{\mathbf{0}}\right)+i \phi_{1}\right] \\
+\delta T_{e d}\left(z_{t}, \mathbf{x}, t\right) \exp (-\eta)
\end{gathered}
$$

where $\delta T_{e d}\left(z_{t}, \mathbf{x}, t\right)$ is the temperature variation associated with eddies at the top of the convection zone, and $\eta$ is the optical depth of the line, used in the observation, at the base of the photosphere.

The summation above is carried out to include all resonant eddies. To project out modes of a particular horizontal wave vector we multiply both sides by $\exp \left(-i \mathbf{k}_{\mathbf{h}} \cdot \mathbf{x}\right)$ and integrate over the area $\mathcal{A}$ of the box. Individual modes are isolated by Fourier transforming in time and the result is

$$
\begin{array}{r}
\delta T_{a}\left(z_{o}, \mathbf{k}_{\mathbf{h}}, \omega\right)=\frac{-\mathcal{A}}{\left(\omega-\omega_{\alpha}\right)+i \gamma_{\alpha}} \sum_{\text {eddies }} F\left(\omega, k_{h}\right) \delta T_{e d}\left(z_{\omega}, \mathbf{x}_{0}, t_{0}\right) \exp \left(i \omega t_{0}-i \mathbf{k}_{\mathbf{h}} \cdot \mathbf{x}_{0}+i \phi_{1}\right) \\
+\tau_{t} z_{t}^{2} \sum_{\text {eddies }} \delta T_{\text {ed }}\left(z_{t}, \mathbf{x}_{1}, t_{1}\right) \exp \left(i \omega t_{1}-i \mathbf{k}_{\mathbf{h}} \cdot \mathbf{x}_{1}+i \phi_{1}^{\prime}-\eta\right)
\end{array}
$$

where the phase $\phi_{1}^{\prime}$ depends on the details of the spacial and temporal structure of eddies, and it should be roughly equal to the phase $\phi_{1}$ so long as the temporal property of the eddy does not change dramatically between the depth where the waves are excited $\left(z_{\omega}\right)$ and the top of the convection zone. 
The observed power spectrum $P(\omega)=\left|\delta T_{a}\right|^{2}$. Assuming that $\delta T_{e d}$ for different eddies are uncorrelated, we retain only those cross terms in $\left|\delta T_{a}\right|^{2}$ which correspond to the same eddy; $\left(t_{0}-t_{1}\right)$ in this case is equal to $\left(z_{\omega}-z_{t}\right) / v_{e d} \equiv \delta t_{t t}$ is the time for downward moving eddies to travel from the top of the convection zone to the depth where they most efficiently excite the mode. The power spectrum at the frequency $\omega_{\alpha}$ is thus given by

$$
P\left(\omega_{\alpha}\right) \approx \frac{\mathcal{A}^{3}}{z_{t}^{2} \gamma_{\alpha}^{3} \tau_{t}}\left|F\left(\omega_{\alpha}, k_{h}\right) \delta T_{e d}\right|^{2}-\frac{\mathcal{A}^{2}}{\gamma_{\alpha}^{2}} F\left(\omega_{\alpha}, k_{h}\right) \sin \left(\omega_{\alpha} \delta t_{t t}\right)\left|\delta T_{e d}\right|^{2} \exp (-\eta)
$$

In deriving the above equation we have assumed that the duration of the observation is greater than the mode lifetime; for smaller observational time $T$, the second term is reduced by a factor of $T \gamma_{\alpha}$.

Due to the multiplicative factor $\sin \left(\omega_{\alpha} \delta t_{t t}\right)$ the second term in equation (16) averages to zero if downward and upward moving eddies contribute equally to wave excitation. Thus the reversal of asymmetry seems to require that downward moving eddies contribute more to the generation of acoustic waves; some recent observations suggest that modes are probably excited by the downflows (cf. Goode et al. 1998).

Comparing the two terms in Eq. (21) with the corresponding terms in equation (2) we find that the phase difference between the observed flux variation of a mode and the turbulent eddy is $\phi=\pi-\omega \delta t_{t t}$; In deriving this result we expanded the Green's function in equation (3) in the neighborhood of an eigenmode: $G_{\omega} \approx \omega\left[\left(\omega-\omega_{\alpha}\right)+i \gamma_{\alpha}\right]^{-1} /\left(8 a \alpha^{2}\right)$. The value of $\delta t_{t t}$ is shown in fig. 4 , and we see that $\phi \sim 1 \mathrm{rad}$ for low frequency modes.

Moreover, from equations (21) and (2) we find

$$
\beta \approx \frac{\omega_{\alpha} \tau_{t}}{8\left(a \omega_{a c}\right) k_{h} z_{t}}\left(\frac{\omega_{a c}}{\omega_{\alpha}}\right)^{2 m-1} \mathcal{M}_{t}^{-3} .
$$

At the top of the solar convection zone $\mathcal{M}_{t} \sim 0.3, z_{t} \sim 300 \mathrm{~km}, g \sim 2.77 \times 10^{4} \mathrm{~cm} \mathrm{~s}^{-1}$, 
$m \sim 1.5, \tau_{t} \sim 100 \mathrm{~s}$, and in the solar photosphere $\omega_{a c} \sim 3.3 \times 10^{-2} \mathrm{rad} \mathrm{s}^{-1}$. The sound travel time from the lower to the upper turning point, $a$, for this model atmosphere is approximately $\omega_{\alpha} /\left(g k_{h}\right)$, and therefore $a k_{h} \sim \omega_{\alpha} / g$. Substituting all this into equation (23) we find the value of $\beta$ to be about 100 for a mode of frequency $2 \mathrm{mHz}$, and this is independent of the horizontal wave number $k_{h}$. A more realistic model of the sun considered in the next subsection yields essentially the same value for $\beta$ when sources are taken to lie at a depth $z_{\omega} \sim 100 \mathrm{~km}$ as suggested by the mixing length theory (see eq. 9). For this source depth the line asymmetry in intensity power spectra is reversed as long as the effective optical depth, at the top of the convection zone, for the wavelength band used in the intensity measurement is less than about 5 so that $\beta^{\prime}=\beta \exp (-\eta)$ is greater than $\sim 0.5$ (see fig. 3).

\subsection{Results for a solar model}

The equation for $\beta$ for a solar model, corresponding to equation (23) of the plane parallel atmosphere, can be derived in a manner analogous to that given in $\S 2.1$ and so we outline a few of the main steps in the derivation.

It is convenient to work with the displacement wave function in this more general case, instead of the enthalpy perturbation used in $\S 2.1$, which we expand in terms of the complete set of eigenfunctions $\boldsymbol{\xi}_{\alpha} Y_{\ell m}$

$$
\boldsymbol{\xi}=\frac{1}{\sqrt{2}} \sum_{\alpha} A_{\alpha} \boldsymbol{\xi}_{\alpha} Y_{\ell m} \exp \left(-i \omega_{\alpha} t\right)+c . c .
$$

The change to the radial displacement amplitude of a mode, due to one eddy, at radius $r_{o}$ in the solar atmosphere is given by (see Goldreich et al. 1993) 


$$
\delta \xi_{r}\left(r_{o}\right) \approx \frac{i \tau_{H} \omega_{\alpha} H_{\omega}^{3}}{2}\left(\frac{\partial p}{\partial s}\right)_{\rho} s_{H} \xi_{\alpha r}\left(r_{o}\right)\left(\frac{\partial \xi_{\alpha r}^{*}}{\partial r}\right)_{r_{\omega}} Y_{\ell m}^{*} .
$$

The notation used here is same as in equation (11). The change to the Lagrangian temperature perturbation in the atmosphere resulting from the excitation due to one eddy is calculated using the above equation and is given by

$$
\Delta T_{\alpha}\left(r_{o}\right) \approx-\frac{i \omega^{2} H_{i} H_{\omega}^{3}}{2(\gamma-1) T_{\omega}}\left(\frac{\partial p}{\partial s}\right)_{\rho} \xi_{\alpha r}\left(r_{o}\right)\left(\frac{\partial \xi_{\alpha r}^{*}}{\partial r}\right)_{r_{\omega}} Y_{\ell m}^{*} \delta T_{e d}
$$

where $H_{i}$ is the pressure scale height at the place in the atmosphere where the optical observation is made, $H_{\omega}$ is the scale length of resonant eddies, and $r_{\omega}$ is the radius where most of the wave excitation takes place. It is straightforward to calculate $\beta$ from this equation

$$
\beta \approx \frac{2(\gamma-1) H_{t}^{2} c_{v}\left(r_{\omega}\right)}{H_{i} H_{\omega}^{3} R_{\odot}^{2} \omega_{\alpha}^{2}}\left(\frac{\partial T}{\partial p}\right)_{\rho}\left[\xi_{\alpha r}\left(r_{o}\right)\left(\frac{\partial \xi_{\alpha r}^{*}}{\partial r}\right)_{r_{\omega}}\right]^{-1} \frac{\omega_{\alpha} \tau_{t}}{a \omega_{a c}},
$$

where the subscript $t$ refers to quantity evaluated at the top of the convection zone, and $a$ is the sound travel time from the lower to the upper turning point of the wave. Figure 4 shows graphs for $\beta$, for three p-modes of a solar model, as a function of depth below the photosphere. The value of $\beta$ for low frequency modes, at reasonable excitation depth, is consistent with our estimate of $§ 2.1$. Also shown in fig. 4 is the eddy travel time, $\delta t_{t t}$, from the top of the convection zone, from which we can estimate the phase difference $\phi=\pi-\omega_{\alpha} \delta t_{t t}$. The values of $\beta$ and $\phi$ for the solar model seem to lie within the parameter range needed for the reversal of line asymmetry.

Specifically, if a mode of frequency $\sim 2 \mathrm{mHz}$ is excited by quadrupole sources lying about $100 \mathrm{~km}$ below the top of the convection zone then $\beta \sim 100$ (see fig. 4); this is same as estimated in $\S 2.1$. For flux variation observations using the central part of the Ni line, one of the filter bands used by the GONG and the MDI instruments, the value of $\eta$, the optical 
depth at the top of the convection zone, is about 6 . Thus $\beta^{\prime}=\beta \exp (-\eta)$ for this source depth and the optical line is about 0.3 , and figs. 3 and 4 suggest that the line asymmtery in the intensity power spectra should be opposite to the velocity power spectra. However, if the acoustic sources are located at a depth of $200 \mathrm{~km}$ or greater, as suggested by Kumar and Basu (1998), then $\beta$ would be much smaller (see fig. 4) and the line asymmetry in the intensity power spectra obtained using the Ni line should be the same as the velocity spectra. The line asymmtery in intensity power spectra, for deeper lying sources, is expected to be reversed when observations are made in the continuum for which $\eta \sim 1$.

The asymmetry reversal requires that the effective observed amplitude of the noise source, $\beta^{\prime} \equiv \beta \exp (-\eta)$, exceed some minimum value of order 0.1 (see fig. 3). Thus we expect the velocity and the intensity power spectra to have the same sense of asymmetry, irrespective of the source depth, in those observations carried out using optical lines formed high up in the chromosphere so that their optical depth $\eta$ at the top of the convection zone is large. This prediction provides a verifiable test of the model discussed here.

For a fixed source depth the value of $\beta$ decreases rapidly with increasing mode frequency (see fig. 4). Thus if modes of different frequencies were to be excited at the same depth, the line asymmetry reversal seen in the intensity power spectra should disappear at some frequency of order $3.5 \mathrm{mHz}$. Asymmetry reversal in the observed spectra at this frequency suggests that the higher frequency modes are perhaps excited higher up in the convection zone.

\section{Discussion}

We find that the observed reversal of asymmetry in the intensity power spectra of solar p-modes can arise as a result of contribution to the observed flux from turbulent eddies that 
excite these oscillations. The flux variations from these eddies is correlated with the p-mode oscillations they generate (as suggested by Roxburgh \& Vorontsov 1997, Nigam et al. 1998), causing a change to the relative contributions of the real and the imaginary parts of the Green's function to the power spectra and consequently to the observed line shape. We have calculated the correlation between the eddy flux variation and the oscillations and find it to be well within the range required to reproduce the reversal in the line asymmetry. The velocity power spectra of solar p-modes are unaffected by the contaminating noise source because the random velocity field in the solar atmosphere, where optical line observations are made, are completely uncorrelated with the oscillations. Intensity power spectra on the other hand contain some contribution from turbulent eddies at the top of the convection zone. These eddies excite solar p-modes as they travel downward, and the flux variation associated with them is correlated with the oscillation they generate.

A reversal of asymmetry in the model problem considered by Roxburgh \& Vorontsov (1997) was obtained when a superposition of dipole and quadrupole sources was considered. However, we find that asymmetry reversal can arise even when modes are excited by quadrupole or dipole sources alone. Furthermore, we have calculated the relative amplitude and the phase correlation in a self consistent manner, and find these to lie in the parameter range that leads to a reversal of asymmetry.

If the model considered here is correct then we expect the asymmetry reversal to disappear when the observed background in the power spectra is vanishingly small. Moreover, the use of spectral lines which are formed deeper in the solar atmosphere, to observe solar oscillations, should have larger amplitude for the flux variation associated with turbulent eddies at the top of the convection zone and hence the line asymmetry reversal should be more dramatic. Conversely observations made in optical lines formed high up in the chromosphere, so that their optical depth at the bottom of the photosphere is very 
large, should show intensity and velocity power spectra to have similar shapes. Recent work of Schou (1998) appears to support this prediction.

We are grateful to John Bahcall for encouraging us to think about this problem, for several helpful discussions, and for his comments on the paper. We thank J. ChristensenDalsgaard for suggesting several improvements to the paper and for mentioned to us the recent work of Jesper Schou. This work was supported by a NASA grant NAG5-7395. SB was partially supported by an AMIAS fellowship. 


\section{REFERENCES}

Abrams, D., \& Kumar, P. 1996, ApJ 472, 882

Christensen-Dalsgaard, J., Rabello Soares, M.C., Rosenthal, C.S., and Thompson, M.J., 1998, in Structure and Dynamics of the Sun and Sun-like Stars, ESA SP-418 eds. S. G. Korzennik and A. Wilson, (Noordwijk: ESA), (in press)

Duvall, T.L.Jr., Jefferies, S.M., Harvey, J.W., Osaki, Y., \& Pomerantz, M.A. 1993, ApJ, 410,829

Goldreich, P., \& Kumar, P. 1990 (GK), ApJ, 363, 694

Goldreich, P., Murray, N., \& Kumar, P. 1993, ApJ, 424, 466

Goode, P.R., Strous, L.H., Rimmele, T.R., Stebbins, R.T. 1998, ApJ, 495, L27

Kumar, P. 1994 ApJ, 428, 827

Kumar, P., \& Basu, S. 1998, submitted

Nigam, R., Kosovichev, A.G., Scherrer, P.H., \& Schou, J. 1998, ApJ 495, L11

Rast, M.P., \& Bogdan, T.J. 1998, ApJ 496, 527

Schou, J., 1998, SOHO preprint

Roxburgh, I.W., Vorontsov, S.V. 1997, MNRAS 292, L33 

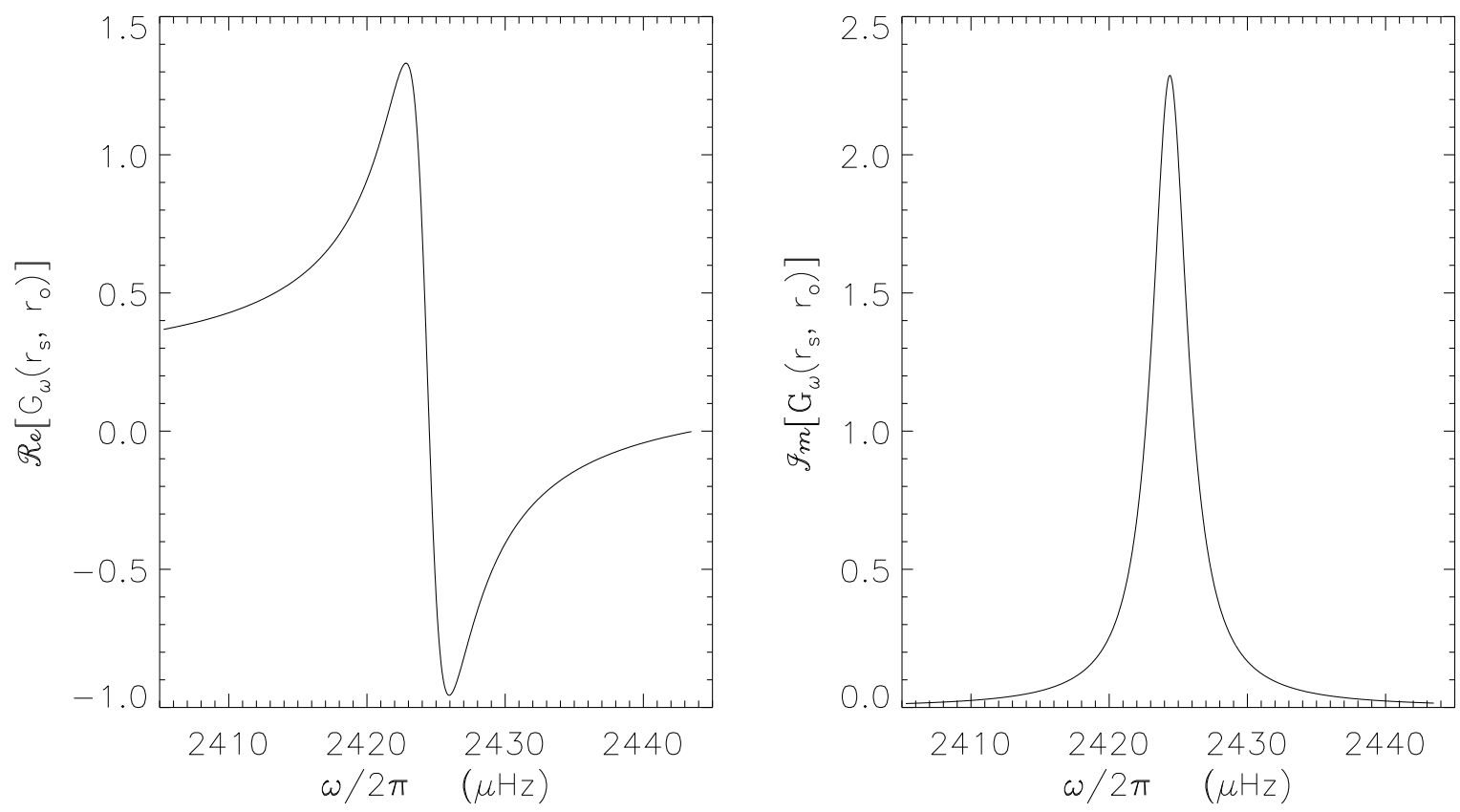

Fig. 1. - The real (left panel) and the imaginary (right panel) parts of the Green's function, for the simple 1-D model problem, at a fixed source and observer location as a function of wave frequency. The source is located in the evanescent region approximately $200 \mathrm{~km}$ above the upper turning point, and the observer is at a place corresponding to the temperature minimum in the solar atmosphere. 

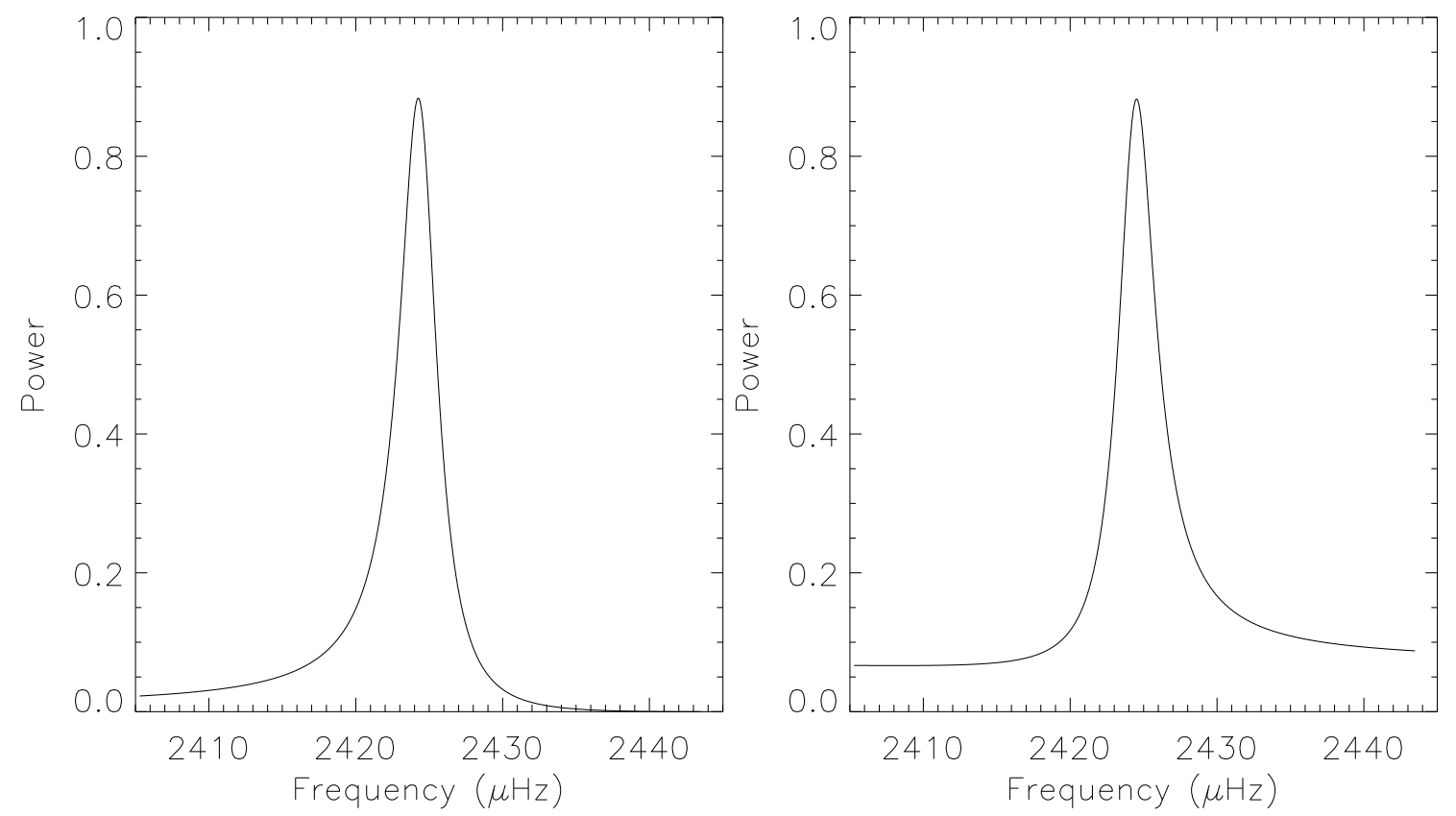

Fig. 2.- The left panel shows the velocity power spectrum for the simple 1-D model problem i.e. it does not include contribution from the noise source. The graph in the right panel includes the flux variation from turbulent eddies and should correspond to the intensity power spectrum; the values of the parameters $\beta \exp (-\eta)$ and $\phi$ are 1.5 and $50^{\circ}$ respectively. 


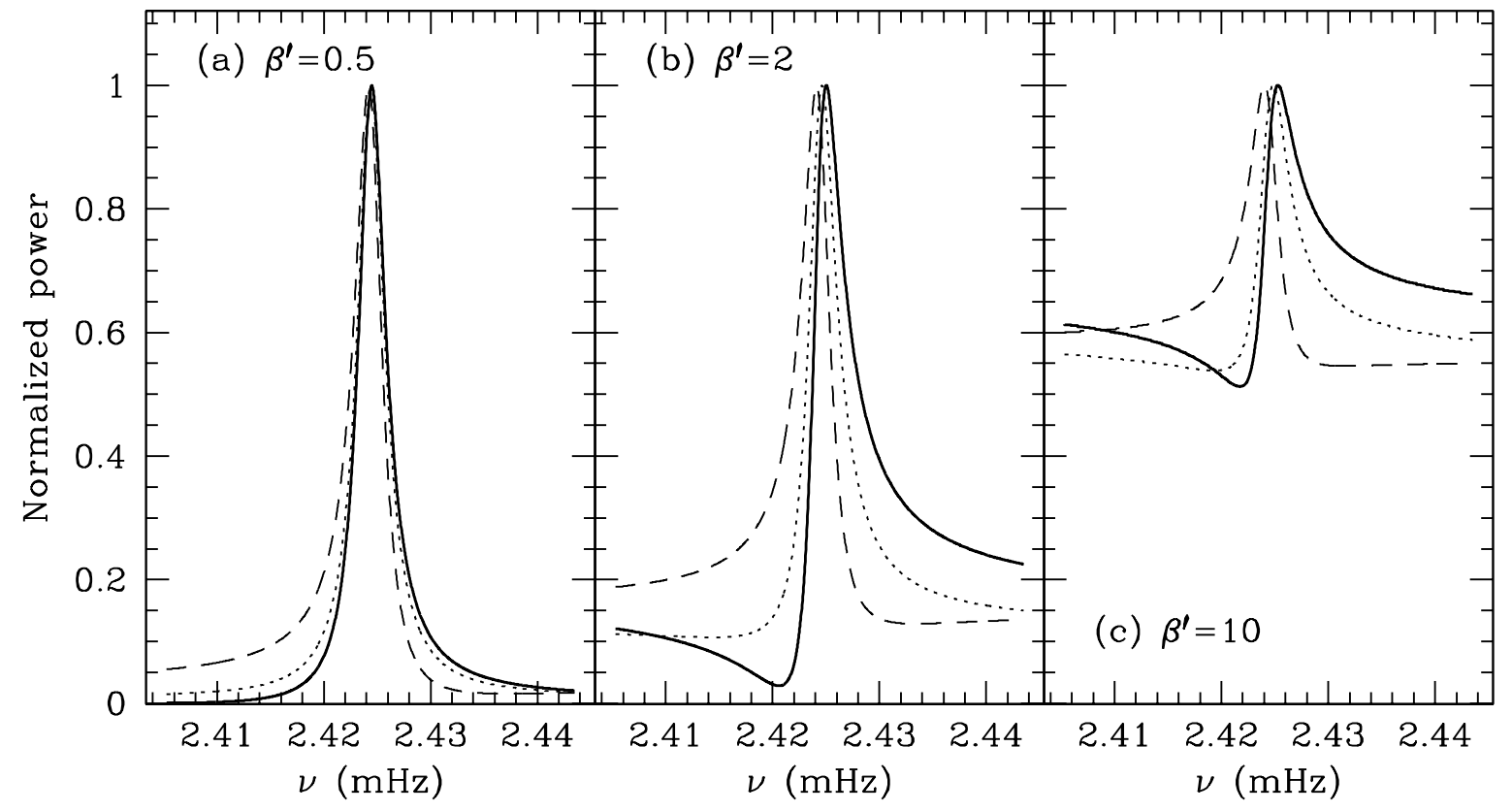

Fig. 3.- Power spectra for several different values of the parameters $(\beta, \phi)$. The continuous lines are line profiles when $\phi=0^{0}$, the dotted lines for $\phi=50^{0}$ and dashed lines for $\phi=120^{\circ}$. For $270^{\circ}>\phi>90^{\circ}$ the sense of asymmetry is same as in the velocity power spectrum (see fig. 2). 

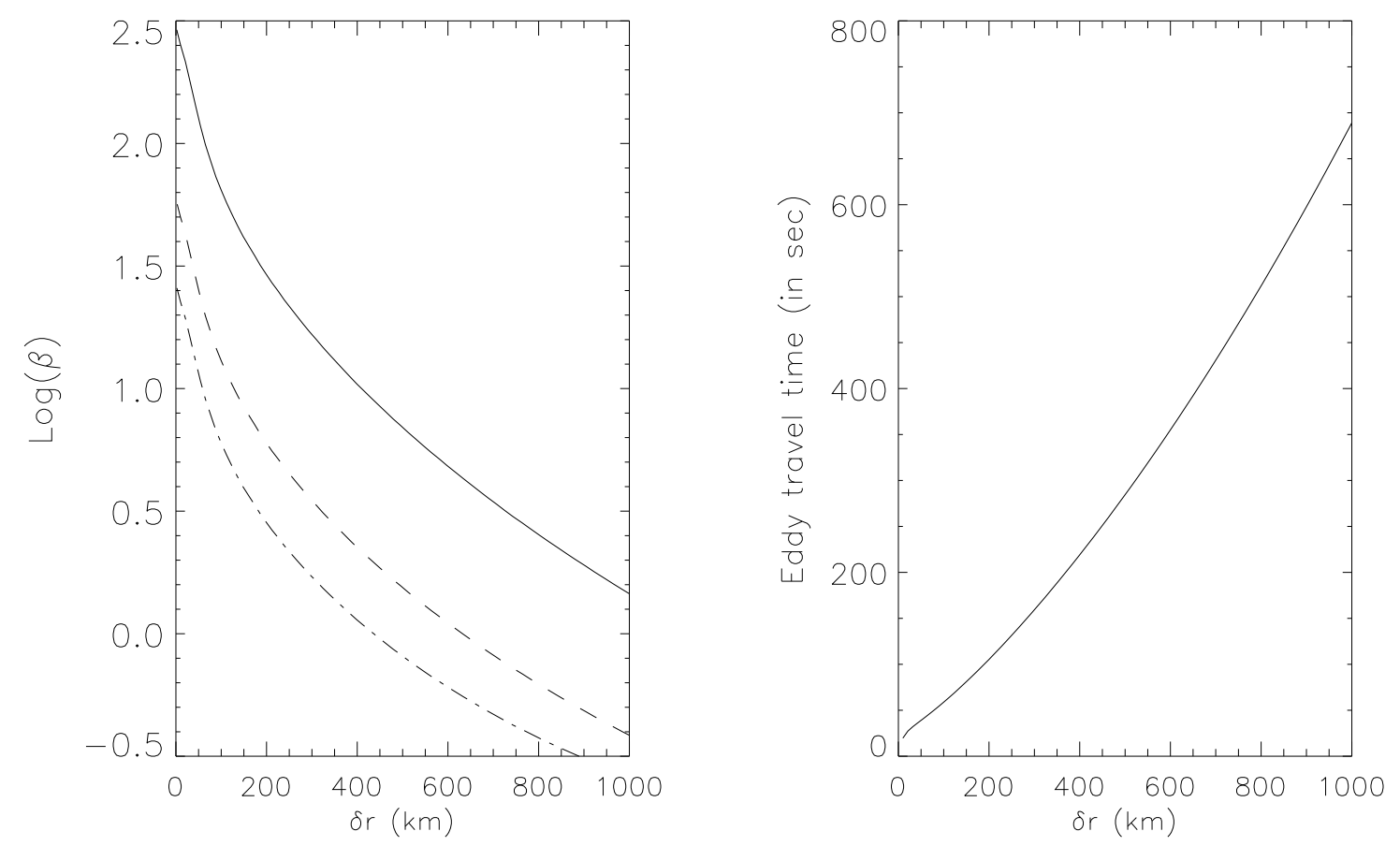

Fig. 4. - The left panel shows the value of $\beta$ for three different modes of the sun as a function of depth measured from the top of the convection zone; the degree $(\ell)$ of all the three modes is 50 , and $\nu=2.08 \mathrm{mHz}$ for the solid curve, $2.67 \mathrm{mHz}$ for the dashed curve and $3.2 \mathrm{mHz}$ for the dot-dash curve. $\beta$ has a very weak dependence on $\ell$; it changes by about $20 \%$ between $\ell$ of 2 and 50 . The panel to the right shows the eddy travel time from the top of the convection zone $\left(\delta t_{t t}\right)$ as a function of depth. 